weicht. Ein anscheineud gutartiger Hydro- oder Haematosalpinx ist z. B. von schweren Netz- und Darmverwachsungen, abgesackten Exsudaten gegen den Douglas'schen Raum begleitet, und ein leicht gedachter Eingriff gestaltet sich dadurch sehr schwierig. Zweitens aber beobachtet man, dass die erwartete Krankheitsform, mit allen Maassregeln zur Klarstellung diagnosticirt, sich nicht findet. Statt der vermeintlichen Geschwulst zeigt sich das normale oder nur entzündlich gereizte Organ lediglich in eine abgekapselte Bauchfellentzündung hineingelagert oder sogar nur in deren Reste und Schwarten eingebettet, ohne dass es zur eigentlichen Geschwulstbildung gekommen íst.

Analysirt man die Vorgeschichte solcher Fälle, so gelangt man für viele derselben nach der Operation zur Erkenntniss, dass mehr die secundären Erscheinungen der chronischen Peritonitis, als die Ursprnngserkrankung zum Siechthum der Leidenden fïhrten, oder dass überhaupt die ursprüngliche Erkrankung (infectiöse Perimetritis, Salpingoophoritis, Perityphlitis) geheilt ist und nur deren Folgezustände nach vielen Heilversuchen zum Bauchschnitte führten.

Aus solchen Beobachtungen und unter Heranziehung der Erfolge der Laparotomie bei Tuberculösen ergiebt sich zunächst ohne Zweifél die Berechtigung, bei allen denjenigen Formen chronischer Bauchfellentzïndung, welche sonstigen Heilbestrebungen trotzen, den Versuch operativer Hülfe zu unternehmen, auch wenn eigentliche Grundursachen ( $z$. B. Tumoren) nicht sicher nachweisbar sind.

$\mathrm{Zu}$ erbringen ist allerdings dann noch der Beweis, dass chirurgisches Handeln wirklich dauernden Vortheil und möglichst völlige Heilung der Kranken nach sich gezogen habe.

Es stehen mir unter 170 Laparotomieen neun Fälle operativen Eingreifens bei chronischer Bauchfellentzündung zu Gebote. Vier derselben warcn tuberculöser Natur und sind kürzlich von Herrn Dr. F. Spaeth veröffentlicht worden; fünf beruhten auf anderen Ursachen. Von sämmtlichen neun wurden fünf, von den uns hier angehenden drei auf Grund irrthümlicher Diagnose in Angriff genommen; bei den letzten beiden wurde auf der Basis der früheren Erfahrung vor dem Eingriffe mit annähernder Sicherheit auf Peritonitis chronica geschlossen. ${ }^{1}$ )

Die ersten beiden sind ein charakteristischer Hinweis, wie vor-

\section{Zur Frage des Bauchschnittes bei Peritonitis chronica.}

Von Dr. L. Prochownick in Hamburg.

Niemand bezweifelt heute nach den Veröffentlichungen der letzten Jahre, insbesondere der sorgsamen Arbeit K ümmell's ${ }^{2}$ ) die Berechtigung des Bauchschnittes bei tuberculöser Peritonitis. Der palliative Nutzen desselben ist so anerkannt, dass er selbst bei bereits vorhandener Lungenerkrankung noch empfohlen wird. Dieser Ausdehnung stimme ich allerdings nicht $\mathrm{zu}^{3}$ )

Auf welchem Standpunkte stehen wir nun gegenüber denjenigen Formen von chronischer Bauchfellentzündung, welche nicht auf tuberculöser Erkrankung beruhen? Bis zu einem gewissen Grade ist allerdings auch diese Frage bereits durch Thatsachen beantwortet. Wenn wir Operationen an entzündlich veränderten Eileitern oder Eierstöcken vollziehen, so bekämpfen wir damit in zahlreichen Fällen noch vorhandene peritonitische Entzündung oder entfernen deren Reste; gleiches gilt für die Ventrofixatio uteri bei verwachsenen Rückwärtslagerungen ${ }^{4}$ ) sowie die kleineren Ovarialgeschwülste. Allein es bleiben noch andere Formen chronischer Entzündung des Bauchfelles übrig, für welche der Nachweis von Berechtigung und Dauernutzen - denn diesen muss man im Gegensatz zur tuberculösen Form beanspruchen - durch chirurgisches Vorgehen noch $z u$ erbringen ist.

Der Erfolg bei der Bauchfelltuberculose hat sich, nach Ausweis der Veröffentlichungen, aus diagnostischen Irrthïmern der Operateure allmählich entwickelt, ähnlich dürfte es uns mit anderen Formen der Bauchfellentzündung ergehen, bis wir zu bestimmter Stellung von Anzeigen für unser Handeln gelangen.

Zwei Beobachtungen werden wohl den meisten, welche häufiger wegen Tuben- oder Tuboovarialtumoren oder kleiner anderer Bauch. geschwülste operirten, nicht entgangen sein.

Die erste besteht darin, dass die Schwere der Nebenbefunde oft in auffälliger Weise von derjenigen der Herderkrankung ab-

1) Meine zum Theil seit 20 Jahren Bestand habenden Krebsheilungen hat Herr Dr. E dw. v. Me yer in Zürich gesammelt und im 28. Band unserer Deutschen Zeitschrift für Chirurgie veröffentlicht; es handelt sich nur um Fălle, deren Diagnose von Eberth in seinen histologischen Cursen geprüft und anerkannt wurde.

2) Archiv für klin. Chirurgie. Bd. 37, p. 1

3) Vergl. den Aufsatz von F. Spaeth; diese Zeitschrift 1889, No. 20

4) Zur Begrenzung des Themas und Verständigung sei bemerkt, dass unter chronischer Peritonitis nur langdauernde Erkrankungen, nicht subacute nach kurz voraufgegangener Infection verstanden sind. Auch die etwaige Beckeneiterungen begleitenden Formen sind in dieser Arbeit von der Betrachtung ausgeschlossen. sichtig die Diagnose auf tuberculöse Bauchfellentzündung zu stell en ist. Nach dem Vorverlaufe und noch bei der Operation rechneten wir sie derselben zu; erst die Untersuchung der entfernten Stücke gestattete, Tuberculose auszuschliessen, und dementsprechend gestaltete sich der spätere Verlauf dauernd günstig.

1. Frau Wlf., Altona. Als Mädchen gesund. Verheirathet Frühjahr 1882. Bald darnach - Gonorrhoe ausgeschlossell, hingegen körperliche Missverhältniss zwischen Mann und Frau - Dyswenorrhoe, Abmagerung. Zunahme der Schmerzen von Monat zu Monat; später auch zwischen den Menses. Gewichtsverlust 25 Pfd. Ende December abendliche Fiebersteigerungen. Aufnahme Anfang Januar 1883. Zwei faustgrosse, prall elastische Tumoren beiderseits von der Gebärmutter. Operation 23. Januar 1883. Ausgebreitete Verwachsung des Netzes mit dem Beckenbauchfell, gelöst, ahgebunden. Blutiger Ascites. Nach Beseitigung einiger Verwachsungen reissen die blossliegenden Geschwülste bei erster Berührung ein, entleeren blutig-seröse Flüssigkeit. Im Grunde derselben beiderseits Eierstöcke, Tuben und Tubenöffnungen normal bis auf geringe entzündliche Auflagerungen. Die pseudomembranösen Tumorwände und ein Theil des Netzes werden entfernt. Auf sãmmtlichen Därmen graue, flockige, schmierige Auflagerungen. Chlorwassertoilette. Bauchnaht. Mehrtägiger Collaps; dann schnelle Gesundung. Frau lebt gesund und blühend, hat geboren (1887!) und genährt. Die Untersuchung der entfernten Stücke zeigte, dass Tuberculose nicht vorhanden war.

2. Fräulein Schdt., Fabrikantentochter, Hamburg. Aufnahme 1. Mai 1886 $16^{1 / 2} \mathrm{Jahr}$ alt. Im 14. Jahre Typhus von 5 wöchentlicher Dauer. Von $14^{1} / 2$ bis zu 16 Jahren regelmässig menstruirt, seit October 1885 nicht mehr. Seitdem schlechtes Befinden, Abmagerung, Kurzluftigkeit, zeitweise rechtsseitige Leibschmerzen; Verdauung und Appetit gut. Befund: Schlankes, zartes Mädchen $(163 \mathrm{~cm}, 45 \mathrm{Kilo})$; flache Brust: anämische Geräusche all Herzen; links Intercostalneuralgie und leichtes pleuritisches Reiben. ${ }^{2}$ ) Rechts im Leibe härtliche, etwas knollige Geschwulst, deren Kuppe etwas oberhalb des Nabels liegt und sich im Bogen zum hig. Poupartii hinzieht; wenig empfindlich; keine Aenderung bei verschiedenen Lagerungen; geringe Verschieblichkeit. Vom Mastdarm lässt sich in Narkose die Geschwilst fühlen, aber nicht abgrenzen; sie scheint adhärent den rechten Gebärmutteranhängen aufzusitzen. Urin gesund. Leistendrüsen beiderseits stark geschwollen. Geschlechtstheile jungfräulich, nicht untersucht, da genügend fühlbar vom Rectum aus. Bei Landaufenthalt bis Anfang Juni bessert sich das subjective Befinden vorübergehend; alsdann Verschlechterung, Gefühl von Vollsein, Spannung; Geschwulst wachsend; Gewichtsabnahme fast 2 Kilo. Unter An-

1) Zwei dieser Fälle sind ziemlich zusführlich bereits früher, diese Zeitschrift 1883, No. 36 und 37 , veröffentlicht worden, beide Krankheitsggeschichten sind daher nur in gedrängtester Kürze gegeben, Einzelheiten betr. mag auf die frühere Publication verwiesen werden. Es sind dieses Fälle I und 3 , in der früheren Arbeit auf Seite 542 u. 544 geschildert.

2) Letzteres rührte von einer Pleuritis her, an welcher sie im Febriar desselben Jahres von Herrn Dr. J. Golds chmid (Altona) behandelt worde war. Bald darnach stellte derselbe die Geschwulstbildung im Leibe fest und hatte die Freundlichkeit, die Patientin an mich zu verweisen. 
nahme einer bösartigen Geschwulst oder Tuberculose Operation am 18. Juni 1886. $\left.{ }^{1}\right)$ Nach Bauchschnitt vom Nabel bis zur Schamfuge zeigt sich ein mit vielen Hunderten von kleinen Knoten besetzter Tumor, wegen dessen bedeutender Grősse zur klareren Uebersicht der Bauchschnitt bis nahe zum Schwertfortsatze ausgedehnt wird. Nach Rückschlagung des mehrfach wach dem Beckeneingang verwachsenen, gleichfalls dicht mit erbsengrossen Knötchen besäten Netzes wird ein Versuch gemacht, die anscheinend colossale Geschwulst zil entwickeln.

Hierbei erst zeigt sich, dass die ganze Masse lediglich aus dem zu einem Convolut vereinten Darmtractus besteht. Vom Duodenum bis zum Rectumanfang bildet der gesammte Darm eine einzige, festzusamınenhängende Geschwulst, in toto am Mesenterium vom hinteren Bauchfell emporzuheben. Nicht eine einzige Darmschlinge ist frei, nicht ein Punkt gestattet irgend welche Unterscheidung. Unzählige kleine Knoten von Erbsen- bis Bohnengrösse, jetzt als Iymphdrüsen kenntlich, überziehen die Geschwulst, lugen aus allen dicht aneinander liegenden Furchen hervor. Der geringste Versuch stumpfer Trennung führt zll starker parenchymatöser Blutung. Der Inhalt des kleinen Beckens ist gleichfalls so allseitig verlöthet und mit denselben Knötchen besetzt, dass eine Unterscheidung einzelner Organe kaum möglich ist. Es wird nach Aufsangung des überall reichlichen, blutig serösen, etwas dickffüssigen Ascites vermittels Chlorschwämmen, ein Stückchen Netz entfernt und eine Serie der Drüsen von verschiedenen Stellen herausgeschält und dann nach sorgsamer Toilette der Leib geschlossen. Der hoffnungslos angesehene Fall gestaltete sich überraschend günstig. Die Genesung von dem Eingriffe vollzog sich ohne jede Reaction, das Mädchen stand nach 14 Tagen auf, nahm von Monat zu Monat zll, entwickelte sich allmählich zur blühenden Jungfrau. Ohne jegliche Störung von Seiten des Darmes, bei regelmässiger Verdauung, bei einer nach 3 Monaten erst schwach, dann immer kräftiger auftretenden, typisch 4 wöchentlichen Meustruation ist die Patientin bisher noch $5 \mathrm{~cm}$ gewachsen, hat sich vorzüglich entwickelt in Ban der Knochen und Muskeln sowie Gestaltung der Brüste, wiegt 65 Kilo, nimmt an allen Vergnügungen ihrer Altersgenossen, sowie an jeder Hausstandsarbeit Theil, und schon haben die Eltern mir die schwierige Frage vorgelegt, wie ich mich zll einer Verheirathung stellen würde.

Die Untersuchung von Netz und Drüsen stellte am frischen und gehärrteten Präparat das Fehlen von Mikroorganismen, insonderheit von Tuberkelbacillen fest. Die Drüsen zeigten auf dem Querschnitte eine centrale, gelbliche Erweichung mit Uebergang in eine härtliche, rosafarbene Randzone; mikroskopisch bestand das Innere aus fettigem Detritus, am Rande war zunächst das Gefüge einer normalen Lymphorüse kenntlich und ging danı olıne sonstige Ausprägung entzündlicher Kernanhäufungen oder Riesenzellen in die fettige, gleichmässige, wenig färbbare Innenmasse über.

An diese beiden Fälle reihen sich drei, deren Endursache in überstandenen acuten Entzündungen des Beckenbauchfells deutlicher charakterisirt war. Bei den letzten beiden wurde von vornherein auf die Wahrscheinlichkeitsdiagnose abgesackter bezw. adhäsiver chronischer Bauchfellentzündung hin operirt.

3. Frau Fck. 34 Jahre alt ${ }^{2}$ ), Arbeiterfrau. 1877 und 78 beiderseitige Parametritis. Nach langer Behandlung Besserung bis Frühjahr 1881. Frische rechtsseitige fieberhafte Beckenbauchfellentzündung mit peritonitischen Reizerscheinungen. Nach Abfieberung wegen Bestehens schmerzhafter rechtsseitiger Geschwulst Punction von der Scheide aus. Schwach eiweisshaltige, klare Flüssigkeit von 1008 spec. Gewicht. 1 Jahr danach Wohlbefinden. Dann Wiederauftreten des schmerzhaften, wenig verschieblichen, jetzt hühnereigrossen Tumors; plötzlich im August 1882 wieder peritonitische Erscheiunngen. September 82 Bauchschnitt. Die Geschwulst besteht aus einem Packete Darmschlingen, verwachsen am Beckenboden und mit zahlreichen Verlöthungen nach den Geschlechtstheilen hin. Zugleich finden sich abgekapselte serōse Exsudate zwischen den einzelnen Schichten der Pseudomembranea und der Serosa uteri. Eierstock und Eileiter gesund. Belassen. Linke Seite gesund. Lösung aller Verwachsungen, Abbindung der unteren mit dem Beckenrande, Darm und Uterns verlötheten Netztheile, sorgsame Blutstillung. Bauchnaht.

Glatte Heilung. Die Frau ist bis Herbst 1886 als gänzlich gesund beobachtet worden. Nur zeitweise klagte sie über Blähsucht und Verstopfung. Seit Herbst 1887 habe ich nichts Sicheres wieder von ihr erfahren konnen, sie soll an Typhus gestorben sein.

4. Frln. Frse., Plătterin, 20 Jahre. Aufnahme 18. August 86. Patientin war stets gesund, menstruirte regelmāssig vom 14. Jahre an, gebar vor ${ }_{3}^{3} / 4$ Jahren leicht ein Mădchen. Năhrte 4 Wochen. Sie will am 3 . und 4. Tage des Wochenbettes Frost und Hitze gehabt haben, stand aber am 7. Tage auf, plăttete am 12. wieder und tanzte bereits in der 4 . Woche ziemlich lange. Gleich nach dem Aufstehen linksseitige Leibschmerzen; dieselben wurden sehr viel heftiger nach dem Tanzen. Lag ohne ärztliche Behandlung 12 Tage im Bett und machte kalte Umschläge. Wieder aufstehend war sie von dieser Zeit an elend, dauernd linksseitige Schmerzen, besonders nach jeder Mahlzeit und jedem T'rinken, Schmerzgefühl vorn Nabel abwärts, Verstopfung mit plötzlichen Durchfällen, die schnell nach den Mahizeiten kommen, wechselnd; unregelmässige Blutungen, dünner gelblicher Scheidenfluss. In letzter Zeit oft Abends Hitze. Mittelgrosse, zarte Person, etwas abgemagert, normale Temperatur, kleiner Puls. Herz und Lunge gut. Urin normal. Leib gespannt, linke Unterhälfte etwas aufgetrieben, auf Druck gering empfindlich. Percussion ohne deutliche Abweichung. Kleine, apfelsinengrosse elastische Geschwulst über der linken Hälfte des kleinen Beckens, ein wenig verschieblich, Gebärmutter etwas nach links gezogen, gegen die Schamfuge gedrängt in Normalstellung. Absonderung der $\mathrm{Ge}-$

1) Bei derselben war Prof. P. F. Mundé aus New-York zugegen und hat selbige in seinem Aufsatze: A glimpse of Laparotomy in Europe, American Journal of Obstetrics etc. Bd. 19, September 1886, erwähnt.

Ebenfalls früher in extenso veröffentlicht, vergl. Bemerkung auf voriger Seite. schlechtstheile arm an Mikroorganismen, insonderheit bei vielen Untersuchungen, auch in der Harnröhre, keine Gonokokken. Die ursprüngliche Annahme einer Tuben- bezw. Tuboovarialgeschwulst wird durch das schărfere Hervortreten der Darmsymptome und bei Narkosenuntersuchung immer unwahrscheinlicher. Bei Jetzterer glaubt man die linke Tube allerdings sicher geschwollen zu fühlen, ebenso den linken Eierstock, aber es macht doch den Eindruck, als wenn die krankhafte Neubildung erst gestielt an demselben såsse. Eine poliklinische Behandlung von über 2 Monaten bringt keinerlei deutliche Besserung. Ende September vermehrte Schmerzen, kleine Fiebersteigerungen Abends, viel Darmerscheinungen. Bei der Operation am 4. October 1886 zeigt sich das Netz über Handbreit mit der linken Tube und der Serosa des Uterus fest verwachsen; unterhalb der Netzverwachsung, mit dem Mesenterium in dicken Strängen an der Hinterwand der Gebärmutter haftend, 2 verklebte Dünndarmschlingen; zwischen denselben unc dem bedeckenden Netze etwa 3-4 Esslöffel blutig-seröser Flüssigkeit abgekapselt. Nach Lösung aller Verklebungen sinken die hochgezogenen linksseitigen Gebărmutteranhänge von selbst abwärts. Ausser lockeren Auflagerungen und starker Röthung durch venöse Blutfülle erweisen sich dieselben gesund. Das Leibende des Eileiters ist offen, secernirt klares Serum, der Eierstock zeigt einen dem. Platzen nahen Follikel, nichts sonst Krankhaftes. Rechte Seite gesund. Sorgsame Säuberung. Unterste Netzpartieen, welche vielfach gequetsclit und stark verdickt sind, werden abgebunden. Glatter Verlauf bis zum Ende der zweiten Woche; in der dritten Woche 8 tägige Fieberbewegung mit Harnverhaltung und einem Bauchdeckenabscess, der von einem Bluterguss zwischen Muskeln und tiefer Fascie ausging. Entlassung am 2. November. Die Operirte blieb bis jetzt gesund, obwohl sie am Sonntage nach der Entlassung die Nacht hindurch tanzte und wahrscheinlich auch sonstigen Schädlichkeiten sich aussetzte.

5. Frau Prhn., Aufnahıne 29. Juli 1887. Bierführersehefrau, 28 Jahre. 5 Jahre verheirathet. Bis zum 18. Jahre regelmässig, schmerzlos menstruirt. Im Anschluss an Typhus wird alsdaun die Regel schmerzhaft und kommt verfrülit. Nach einem schweren Scharlach kurz vor der Verheirathung gesellen sich dazu häufige rechtsseitige Leibschmerzen. Nach der Verheirathung werden dieselben schlimmer, die Cohabitation ist stets schmerzhaft. Vor zwei Jahren war durch Discision Besserung erstrebt worden mit vorübergehendem Erfolge. Befund: Geschrumpfte rechtsseitige Para- und Perimetritis, Herabsenkung des rechten Fierstockes ohne wesentliche sonstige Veränderung, geringe, steile Retroversion mit Rechtslagerung durch Narbencontraction. Bis Ende September 1887 bedeutende Besserung durch eine örtliche Massagekur mit gleichzeitigen Sitzbädern, vaginalen Jodpinselungen und Glycerintampons. Anfang October im Anschluss an einell rohen Coitus des betrunkenen Mannes frische, rechtsseitige Parametritis mit geringem Fieber. Nach Schwund der acuten Erscheinungen bleibt die Frau elend, klagt über Magenschmerzen nach jedem Essen oder Trinken mit sofort folgendem Schmerz rechts unten in Leibe und Blasendrang; beim Aufstehen und Gehen macht ihr jeder Schritt Beschwerden, sie fühlt die oberen Theile des Leibes so nach unten gezogen, dass sie vornübergebeugt gehen muss und fortwährend einen Halt in der Nabelgegend haben möchte. Dabei lästige Flatulenz mit plötzlichen kurzen Durchfällẹn verbunden, die besonders warmen Getränken folgen. Zur Zeit der verstärkt und verfrüht auftretenden Monatsblutung steigern sich diese Beschwerden zur Unerträglichkeit. In der rechten Seite findet sich oberhalb des frischen, allmählich schwindenden Exudates im Beckenbindegewebe ein zweiter, früher nicht nachweisbarer im Bauchfell sitzender, überapfelgrosser, elastischer Tumor, der weder dem Eileiter noch dem Eierstocke angehört, da es möglich ist, beide nach Maassgabe des von früher bekannten Befundes abzutasten. Nachdem weder langes Liegen, noch sonstige Behandlung irgend eine Besserung im Gefolge gehabt hatte, wurde auf dringende Bitte der Frau mit der Wahrscheinlichkeitsdiagnose einer abgekapselten Pelviperitonitis chronica dextra und starker Netzbeckenverwachsungen am 12. Januar 1888 operirt. Der Befund war der vom vorigen Falle auf die rechte Seite übertragell. Nur erstreckte sich die Netzverwachsung noch auf das Peritoneum parietale des vorderen Beckenrandes. Eierstock und Eileiter lagen nach Lösung der Anheftungen völlig frei, und die Tube war geöffnet, mit normalem Secret. Ausserhalb des Bauchfelles konnte man ein derbes Infiltrat im rechten Beckenbindegewebe durchfühlen; auch hier sanken nach Lösung des Netzes die Genitalien sofort in die Tiefe, und die vorher deutlich steile Rückwärtsneigung des Uterus äuderte sich sofort zu einer normalen Anteflexion. Linksseitige Gebärmutteranhänge völlig gesund. Beendigung der Operation wie im vorigen Falle. Glatter Verlauf. Völlige Genesung; das parametritische Exsudal heilte glatt während des Liegens nach dem Eingriffe, alle peritonitischen Reizerscheinungen verschwanden; seit einiger Zeit ist sie nach 8 jähriger Unfruchtbarkeit schwanger, und es fehlen auch in diesem Zustande alle Beschwerden, welche anf irgendwelche Bauchfellreizung deuten könnten.

Von diesen 5 Fällen steht der zweite für sich allein. Besässen wir nicht die sichere Begründung von Tuberculose im bacteriologischen Nachweise, so würde derselbe sicher derselben zugezählt und könnte dann als Heilung glänzen. Aber auch nachdem dies durch negatives Ergebniss der Untersuchung ausgeschaltet ist, bleibt er noch dunkel genug. Ob die Ursprungserkrankung in dem früheren Typhus oder einer mit multiplen Drüsenschwellungen einhergehenden Erkrankung (Scrofulose?) zu suchen sei, oder, was uns bei der Annahme von Tuberculose am nächsten lag, von der kürzlich überstandenell Pleuritis abhing, ist nicht zu entscheiden. Ausser Zweifel steht die günstige Einwirkung, welche durch den Eingriff auf die Bauchhöhle geübt wurde; ob wir sie der Entleerung des Ascites oder der Lostrennung des in breiten Flächen mit dem Beckenbauchfell verwachsenen Netzes zuschreiben wollen, bleibe fraglich; nach Vergleich mit den anderen Krankheitsfällen möchte ich persönlich ınich für das letztere entscheiden. An dem späteren Ver- 
lanfe ist ueben der völligen Regelnug der Geschlechtsfunction vou besonderem physiologischem Interesse, dass niemals irgendwelche Störnngen in der Darmthätigkeit eingetreten sind, - anch vorher waren dieselben gering - obwohl doch nicht anznnehmen ist, dass die vollständige Verwachsung des Gesammtdarmes zn einer Masse sich später wieder gelöst habe.

Anch Fall 1 wnrde zuerst für Tnbercnlose angesehen; für diese Aunahme bot die Krankengeschichte hinreichende Gründe; die Untersuchung der entfernten Netzstücke belehrte nus eines Besseren, nud der weitere Verlanf hat bewiesen, dass wir es unr mit einem Falle von Peritonitis chronica $z n$ thun hatten, welcher bis anf einen geringen bald $\mathrm{zn}$ berührenden Unterschied den ïbrigen Fällen gleicht.

Zwei Gesichtspnnkte sind es vornehmlich gewesen, welche mich bewogen, diese an Zahl kleine Reihe von Beobachtungen zn veröffentlichen, einmal nämlich die Art nnd Ursache der Erkranknug, zweitens der Weg und Erfolg der Behandlnng. Ansser Zweifel steht, dass in den 4 Krankheitsfällen (1. 3. 4. 5.) der Ansgangspnukt vou den Geschlechtsorganen zu suchen ist; einmal unr (Fall 1) fehlt eine vorhergegangene acnte Krankheit, dreimal ist sie nachweisbar. Nnn ist gonorrhoische Anstecknng znnächst bei allen vieren anszuschliessen. Die Mänuer wurden nicht nur befragt, sonderu anch untersucht. Bei den Patienten selbst war weder ans der Vorgeschichte, noch ans den Befunden ein Hinweis dafür zn entnehmen. Ferner charakteristisch ist, dass in der Symptomenreihe sowohl, als im Befund ansgesprochene Zeichen von Gebärmntter-, Eileiter und Eierstockserkranknug fehlten. Weder typische Wehenoder Eierstocksschmerzen, noch heftigere Endometritiden, noch nuregelmässige stärkere Blntverlnste waren vorhanden; geringe Uterinabsonderung nud Dysmenorrhoe dürfen wohl als gewöhnliche Begleiterscheinnngen pelviperitonitischer Reiznng aufgefasst werden. Weiterlin fehlten bei den Kranken ansgesprochene Lendenmarksymptome, hysterische Reflexe an entfernteren Körperstellen nud psychische Erscheinnngen, wie sie in allerdings verschiedenem Grade der Oophoritis, besonders aber der Salpingitis chronica bezw. den Tnboovarialtnmoren, zn eigen sein pflegen.

Dieses alles znsammengenommen berechtigt $\mathrm{zu}$ der Anschannng, dass bei nuseren Kranken die Pelviperitonitis von Erkranknng des Beckenbindegewebes ans (Infection anf lymphatischem Wege) ${ }^{1}$ ) ihren Ursprung genommen habe. Oder aber, selbst angenommen, die Eingangspforte sei Gebärmntter nud Tube gewesen, so müssten bestimmte Entzïndnngserreger diese Organe selbst gar nicht oder nur vorübergehend schädigen nnd erst im Banchfelle zn einer verderblicheren, danernden Einwirknng gelangen. Denn wir fanden die Eileiter gesuud, am abdominalen Ende geöffuet, normal absonderud, an den Eierstöcken unr leichte entzündliche oder fibrinöse Anflagernngen; dies steht im direkten Gegensatze zn allen Beobachtnngen, welche wir bei der direkten Infection durch Uterns nud Tube abseiten der bekannten pathogenen Entzündnngserreger zu machen Gelegenheit haben.

Es müssen also entweder gleiche Entzündnngserreger, je nachdem sie ihren Weg nehmen oder primär localisirt werden, verschiedene Formen von Beckenbanchfellentzündnng Pelviperitonitis erzengen können, oder es müssten diese verschiedenen Formen anch ganz verschiedenen Krankheitsträgern ihre Entwickelnng verdanken. Für acnte Erkrankung, besouders im Pnerperinm, sind wir bereits zu der Erkenutuiss gelangt, ${ }^{2}$ ) dass beide Möglichkeiten vorliegen; in der Gynäkologie stehen wir meist chronischen Krankbeitsprocessen gegenîber und müssen versuchen, den mangelnden bakteriologischen Nachweis durch Zerlegnng der Vorgeschichte nnd der Einzelgruppen von Krankheitserscheinungen bei jedem Falle zn ersetzen.

Dabei erscheint es vor allem wichtig, die entweder gar nicht von Uterns nud der Tube ans das Bauchfell erreichenden Entzündungen von denjenigen zn trenuen, welche direkt ans der Tube ïbertragen werden; zn einem solchen Versuche schienen mir unsere Fälle eine gewisse Anleitnng zn geben; zu einem bestimmteren Urtheile wird es freilich erst noch weiterer Beobachtnngen bedürfen.

Neben der gemeinsamen Entstehnugsweise der Peritonitis, in nuseren Fällen mit wahrscheinlicher Umgehung des Uterus und der Tnbe als Eingangspforte, bieten dieselben ein anderes gemeinschaftliches klinisches Bild in ansgesprochener Form, welches meines Erachtens bisher in der Gynäkologie bezw. Chirnrgie nicht genügend gewürdigt worden ist, ich meine die Verwachsungen des grossen Netzes mit den Organen des kleinen Beckens. Das, wenigstens in seinem grösseren nnteren Theile, ans vier Banchfellplatten bestehende

1) Anch an rein tranmatische Ergüsse kann dabei wenigstens für Fall 1 gedacht werden, bei dem jedes nrsächliche Moment ansser einem starken Missverhältnisse in der Grösse der Geschlechtsorgane von Mann nnd Fran fehlte.

2) Man vergleiche die bacteriologischen Arbeiten über das Pnerperalfieber in den letzten Jahren, welche sowohl verschiedene typische Mikroorganismen (Staphylokokken, Streptokokken, Erysipelkokken), als jeweilige alleinige Erreger ergeben, als auch das Eindringen anf verschiedenen Wegen, z. B. anch mit völliger Urngehnng von Uterus nnd Tube, sicher nachweisen. nud deshalb für adhäsive Entzündnug sehr geeignete Netz ist in der Chirnrgie der Brüche und Banchwunden genïgend bekannt und gewürdigt; dass es bei seiner grossen Bewegnngsfreiheit weithinnnterreichenden Lageverändernngen nuterliegt, lehrt uns neben der Beobachtnng am Sectionstische anch diejenige am Krankenbett. Denn manche Kranke mit rapid eintretender Pelviperitonitis verdankt sicherlich die Erhaltnug ihres Lebens einer schnellen adhäsiven Entzündung und dadurch entstehenden Abkapselnng von SiSeiten des grossen Netzes. Non liegt aber anf der Hand, dass dieser grosse Werth des Netzes für die Entzündnngsvorgänge im Banchfelle anch seine Schattenseite haben muss. So lange die Kranken liegen, giebt sich dies nicht knnd; aber bald nach dem Anfstehen nnd gerade mit der Resorption der angenblicklichen Krankheitsprodncte treten nuangenehme Erscheinnugen ein, welche daranf beruhen. dass dem Netze seine freie Beweglichkeit genommen ist. Wie bei dei Betheilignng von Netz an Leisten- und Schenkelbrüchen, klagen die Franen in der Convalescenz nach Beckenbanchfellentzündnng über Uebelkeit, Erbrechen nnd ziehende Schmerzen vom Magen abwärts, besonders bei Anfüllung des Magens, also nach dem Essen, nnd am meisten nach warmen, die Peristaltik stark anregenden Getränken.

$\mathrm{Zn}$ diesen Empfindnngen gesellen sich Spannung im Leibe, Gefiihl von Auftreibung nud Blähsucht. Je nachdem im Becken eine Verwachsung mit den Eingeweiden besteht, gestalten sich die Symptome. Es treten sowohl Blasendrang, ziehende Schmerzen nach dem Uriuiren, als Stnhldrang ohue Erfolg, oder Wechsel von Dnrchfall mit hartuäckiger Verstopfung ein, je nachdem die Netzmassen an Blase, Darmschlingen, oder Rectnm bezw. Flexur angeheftet sind. Die Verwachsnngen mit den Geschlechtsorganen geben nicht so ansgesprochene Erscheinnngen. Jedoch gestatten anch hier einzelne Angaben, anf Netzverwachsung zu schliessen. Wenn bei fiüherem Fehlen ähnlicher Erscheinnngen nach überstandenen Beckenentzündnngen über ein fortwährendes Ziehen vom Nabel nach der Schamfnge abwärts geklagt wird, wenn einzelne Kranke darnach sogar ibre Körperhaltung einrichten, wenn bei längerem Gehen nnd besonders Abwärtssteigen (Treppen) Magendrnck nud Uebelkeit eintritt, die sonst regulären Menses mit Cardialgieen verlanfen, so liegt der Schlnss einer Netzgenitalverwachsnng nahe nud wird, wenn vou Seiten der Blase oder des Darmes Symptome, wie oben geschildert, zngleich vorliegen, zur Sicherheit ${ }^{1}$ ). Dass diese Beschreibnng keine theoretische sei, lässt sich am Operationstische mit fortschreitender Erfahrung klar beweisen. Einmal anf diesen Znsammenhang anfmerksam geworden, kann man allmählich die Verwachsungen des Netzes und die Oertlichkeiten derselben nit einer mitunter verblüffenden Bestimmtheit voranssagen, wie es mir in den letzten Jahren bei vielen Salpingotomieen nnd Entfernnug von Hämatovarien oder Pyovarien ergangen ist. Noch charakteristischer kann sich die Vorhersage bei solchen Franen gestalten, welche znm zweiten Male einem Banchschnitte nnterzogen werden müssen.

Verwachsungen des Netzes mit dem Peritonenm parietale finden sich bei solchen geradezn als Regel, solange aber kein Banchbrnch vorhanden ist, machen sie kanm je Erscheinungen. War aber der Verlanf nach der ersten Operation kein glatter, waren viele Verwachsungen zn lösen, waren nach derselben Exsudate oder Stielentzündnngen mit peritonitischer Reizung nnd Fieber vorhanden, so stellen sich bei diesen Leidenden fast stets einzelne Symptomgruppen der Netzbeckenverwachsung ein, welche man bei einem wiederholten Eingriff voranssagen kann nnd bestätigt findet. ${ }^{2}$ ) Bei den meisten Kranken nnn heilen diese Krankheitserscheinnngen, sowohl nach Entzündungen, als nach Operationen, d. h. sie werden symptomlos. Von einer eigentlichen Heilung kann dabei kanm die Rede sein, allein entweder dehut sich das Netz mehr und mehr, oder der Patient gewöhnt sich an die Empfindnng; das Bestehenbleiben der Verwachsnng erweist danu mitnuter nach späterer anderweitige Erkranknng die Section. ${ }^{3}$ ) Bei einigen aber werden die Netzbecken-

Allerdings kommen bei schweren verwachsenen Retroflexionen ähuliche Erscheinungen vor; allein bei der chronischen Pelviperitonitis finden sich vorwiegend steile Retroversionen der Gebärmutter. Anch baben sich mir bei den Ventrofixationen solcher Gebärmntter - bisher 11 eigene Beobachtungen - nahezu ausnahmslos zngleich Netzbeckenverwachsungen ergeben, weun entsprechende Symptome den Fall complicirten.

Vergl. nnten p. 478. Mir selbst stehen darn̈ber vier Beobachtnngen zn Gebote; zwei Mal Ovarialgeschwn̈lste der früher zurückgelassenen gesunden Seite - glatte Operationen das erste Mal, lediglich Netzbanchwandverlöthnngen ohne Symptome bei dem zweiten Banchschnitte; je ein Mal Castrátion und einseitige Salpingotomie mit nicht ganz glattem Verlanfe, dabei beträchtliche Netzbecken- bezw. Darmverwachsungen beim zweiten Nale, deren Isocalisation ziemlich genan vorher bestimmbar war.

) Es bot sich uns einige Male Gelegenheit, Kranke mit solchen Klagen nach Pelviperitonitis später zn seciren nud die Verwachsungen des Netzes festzustellen; am charakteristischsten war ein Fall mit pelviperitonitischem Exsudat nnd späterer Netzadhäsion entsprechenden Klagen nach Totalexstirpation von der Scheide aus; Tod nach 15 Monaten an Recidiv. Starke Netzverwachsung mit dem Beckenbanchfell über der Operationsnarbe. 
verwachsungen zu einer Ursache dauernder, ansteigender, schliesslich die Arbeitsfähigkeit und das Leben bedrohender Beschwernisse. Es hat mir den Eindruck gemacht auf Grund unserer Fälle, als wenn die Breite der Verwachsung mit dem. Beckenbauchfell und das Vorhandensein abgekapselter Exsudate zwischen Netz und einem der Organe im kleinen Becken von besonders erschwerendem Einflusse wäre. Oefters führt die Nutzlosigkeit längerer Heilbestrebung schliesslich zum chirurgischen Eingriff, um so mehr, als die Exsudate kleine Geschwülste vortänschen, welche für tubaren oder ovarialen Ursprunges angesehen werden. Die Operation führt zunächst zu einer Erkenntniss des diagnostischen Irrthums, dann aber zu der wichtigeren Frage, wieweit soll sich nun das chirurgische Handeln erstrecken.

Und damit gelangen wir zu dem zweiten Gesichtspunkte, von welchem ausgehend diese Fälle publicirt wurden. Es kaun nach der Betrachtung unserer Krankheitsfälle nicht zweifelhaft sein, dass auch Entzündungen des Beckenbauchfelles zu chronischer Peritonitis mit Bildung von abgesackten Ergüssen fïhren können, ohne dass Gebärmutter und Eileiter einen mehr als ganz secundären Zusammenhang mit dem Krankheitsprocesse haben, und ohne dass die Eierstöcke in irgend einer hervorragenden Weise an demselben betheiligt zu sein brauchen. Der Versuch, bei solchem Befunde, wenn er einmal zum operativen Vorgehen gedrängt hat, möglichst organerhaltend" zu verfahren und sich lediglich auf die Beseitigung der Peritonitis chronica und ihrer Erzeugnisse zu beschränken, ist als durchaus gelungen $z u$ bezeichnen. Als ich mir bei dem ersten Falle weder über die Entstehung noch über den Zusammenhang dessen, was sich bei der Laparotomie darbot, klar war; bedurfte es einer längeren Ueberlegung, die Eierstöcke, welche nur einige fibrinöse Anflagerungen zeigten und nach Abtupfen mit einem Schwamme dann ganz glatte Oberfläche hatten, sowie die Tuben im Leibe zu lassen; die Schwere der Netzverwachsung, die Entleerung des Beckens von abgesackter Flüssigkeit, das jugendliche Alter der Frau brachten mich zu dem Entschlusse, den Versuch zu machen, wieweit eine Genesung ohne Castration erreichbar sein wïrde. Der wider Erwarten günstige Verlauf bestimmte mein Vorgehen für die übrigen Fälle. Die Kranken sind nicht nur ohne Rückfälle geheilt, sondern zwei derselben haben auch nach längerer voraufgehender steriler Ehe und unter Verhältnissen, welche ohne die Operation eine Empfängniss durchaus unwahrscheinlich machten, concipirt. Alle, in noch verhätnissmässig jugendlichem Alter stehend, sind vor der Invalidisation durch Castration bewahrt worden.

Es darf auf Grund dieser Beobachtungen wohl emp fohlen werden, sowohl bei chronischer Peritonitis, besonders wenn sich ausgebreitete Netzverwachsung erkennen lässt, nach Erschöpfung anderweitiger Behandlung die Heilung durch Bauchschnitt zu versuchen, als auch beim Befunde chronischer Peritonitis an Stelle erwarteter Eileiter- oder Eierstockserkrankung während einer Operation, sich lediglich mit der Beseitigung der Entzündung und ihrer Reste zu begnügen, ohne die Organe selbst zu entfernen. In letzterem Falle hat man sich von der Beschaffenheit der Eierstöcke durch Besichtigung derselben, bezw. Follikelpunction, besonders aber von derjenigen der Tuben durch Herauspressen von Secret aus dem auf seine Durchgängigkeit zu prüfenden Bauchende zu überzengen. Enthält die Tube Eiter, so ist die Salpingotomie vorzunehmen.

Ueberblicken wir, nach ähnlichen Erfahrungen und darauf gegründeten Anschauungen suchend, die Litteratur der letzten Jahre, so ist die direkte Ausbeute gering. Neben zahlreichen Berichten über Operationen bei acuter Bauchfellentzündung verlautet über die chronische Form - ausgenommen die tuberculöse - als mit Absicht in Angriff genommen, nichts. Von den Berichten über tuberculöse Peritonitis gehören allerdings meines Erachtens wohl manche mehr in die Kategorie solcher Fälle, wie wir sie unter Fall 1 und 2 oben kennen gelernt haben; sonst aber finde ich nur noch 2 Fälle, bei denen man an Stelle anderer Erkrankung lediglich chronischer Peritonitis begegnete. ${ }^{1}$ ) Hingegen finden sich in den Einzelberichten über grössere Reihen von Bauchoperationen hăufig wiederkehrend unvollendete Operationen beschrieben, bei denen ohne Organentfernung nach Beseitigung von Netz- und anderen Verwachsungen Heilung eintrat; ähnliche werden in Verhandlungen von Fachgesellschaften berichtet, und in den Debatten derselben kann man einen Umschwung der Anschauungen deutlich verfolgen. Derselbe zielt dahin, dass bei den zur Castration und Salpingotomie bestimmten Krankheitsfällen der begleitenden Bauchfellentzündung eine wichtigere Rolle zukommt, als man bisher' annahm, und dass man mit Rücksicht darauf mehr auf organerhaltende Operationen hinzustreben habe.

J) Valerani, Gazz. delle cliniche 1885, No. 21. Jelaew, Medicinische Rundschau, (Russisch) 1886, No. 11. Beide Arbeiten habe ich nur in verschiedenen Referaten, nicht im Original erhalten können.
Ohne auf diese zerstrenten Berichte näher einzugehen, vergegenwärtigen wir uns, dass eine der ersten Anregungen von Karl Schröder ${ }^{1}$ ) ausging, welcher bei Ovariotomieen verschieden grosse Stücke gesunden Eierstocksgewebes in der Bauchhöhle zurückliess, um die Menstruation bezw. Möglichkeit der Empfängniss zu erhalten. Sein genialer Gedanke war von Erfolg gekrönt.') Neben ihm gebührt vor allen A. Martin das Verdienst, bei seinen Operationen möglichst organerhaltend verfahren und dieses Vorgehen begründet $z u$ haben, besonders für Eingriffe bei kleineren Beckengeschwülsten. Auch er hält für die Mehrzahl der Fälle chronischer Oophoritis die umschriebene Bauchfellentzündung für das entscheidende ursächliche Moment $;^{3}$ ) dasselbe macht er für die Tube geltend und empfiehlt daher bei Operation einer kranken Seite mit der anderen, unter eventueller Beseitigung peritonitischer Fntzündungsreste, möglichst organerhaltend $\mathrm{zu}$ verfahren. Er hält dies sogar trotzdem aufrecht, dass er sich mehrfach - allerdings bei Gonorrhoischen - za zweiten Eingriffen gezwungen sah.

Nun ist es aber von der Zurücklassung einer gesunden Seite bei Ausschneidung der anderen erkrankten, bis zur Erhaltung beider Seiten, wenn sie nach Entferoung der umschriebenen Banchfellentzündung und ihrer Reste als gesund erkannt werden, nur ein einziger Schritt! Derselbe bindet sich an die Voraussetzung, dass die Beckenbauchfellentzündung entweder nicht von Uterus und Tube ausging oder aber dieselben nicht dauernd veränderte, bezw. dass die Entzündungserreger in denselben, besonders der Tube, nicht mehr vertreten sind. Darüber lässt sich durch Untersuchung bei der Operation (cf. oben) und Berücksichtigung der Vorgeschichte (Ausschliessung gonorrhoischer Ansteckung) hinreichend Aufschluss erlangen, nm darauf ein organschonendes chirurgisches Handelı zu begründen. Die Richtigkeit desselben unter diesen Voraussetzungen wird durch unsere Fälle erwiesen.

Aus der an den Martin'schen Vortrag, welcher oben citirte Ansichten enthält, anschliessenden Discussion ${ }^{4}$ ) geht aber noch einiges andere für unsere Anschauungen Wichtige hervor. Die an selbiger betheiligten Operateure betonen sämmtlich, wie bedeutenden chronisch peritonitischen Veränderungen sie gelegentlich zweiter Laparotomieen begegneten, und zwar selbst nach einfachen, noch mehr freilich nach schwierigen oder im Heilverlaufe complicirten ersten Operationen. Der Netzverwachsungen wird dabei - besonders in den Martin'schen Krankengeschichten -- Erwähnung gethan. Die Wichtigkeit dieser Veränderungen für die Symptomatologie und Beurtheilung fernerer Operationen wird betont. Dies lehrt zweierlei. Einmal weist es darauf hin, wie wir auch bei ersten Operationen uns bemühen müssen, die Krankheitserscheinungen zu zerlegen in diejenigen, welche von eigentlichen Erkrankungen bestimmter Organe (Genitalien, Blase, Darm) ausgehen, und diejenigen, welche lediglich auf bestehende oder abgelaufene Bauchfellentzündungen bezw. deren Folgezustände zu beziehen sind. Die solcher Erkenntniss entgegenstehenden Schwierigkeiten sind nicht geringe, allein sicherlich mit der Zeit überwindbar, wenn immer zwischen dem Operationsbefund und der Krankengeschichte eine nachträgliche Parallele gezogen wird. Zweitens aber liegt in obenerwähnten Erfahrungen die Aufforderung, bei den ersten Operationen so weit als möglich der Bildung chronischer peritonitischer Entzündungszustände und deren Folgen - Darm- und Netzverwachsungen - entgegenzuarbeiten. Dies geschieht neben anderen wichtigen Momenten, z. B. Uebernähen breiter, succulenter Stiele, Vermeidung von Brandschorfen, Enthaltung von Opiaten vor und nach der Operation, in erster Linie durch genügende Entfernung aller bereits bestehenden chronischperitonitischen Veränderungen. Flottirende Stränge werden mit Catgut abgebunden, leichtere Darmverklebungen gelöst, gequetschte Netztheile entfernt, bezw. überlanges, verdicktes Netz gekürzt, stärker adstringirende antiseptische Flüssigkeiten vermieden; langdauernde Bauchtoiletten, besonders Donglasreinigungen, umgehe man wegen der vielen damit verbundenen mechanischen Epithelabschürfungen thunlichst. Für einzelne Operationstypen, in erster Linie die Salpingotomie - in specie die einseitige - ist die Beobachtung dieser Regeln für den späteren Verlauf von besonderer Wichtigkeit und darf auch nicht, um heim Operiren Zeit zu sparen, geopfert werden.

Dass die verwendete Sorgfalt sich lohne, geht auch hier aus dem dauernden Heilerfolge bei unseren Kranken hervor.

1) Zeitschrift für Geburtshülfe und Gynäkologie XI. p. 355 .

2) Es ist von ganz besonderem Interesse, dass eine der Schröder. schen Kranken (doppelseitige Ovariotomie mit partieller Organerhaltung) einer zweiten Operation durch Herrn J. Veit unterzogen werden musste (Zeitschrift für Geburtshülfe und Gynäkologie XV, p. 291) und dabei gerade eigentlich lediglich chronische Peritonitis ur die rechten Adnexa herum, ohne deutliche Organerkrankung, festgestellt wurde.

3) Zeitschrift für Geburtshülfe und Gynäkologie XV, p. 269--271.

4) Zeitschrift für Geburtshülfe und Gynäkologie XV, p. $287 \mathrm{ff}$. 